\title{
EFFECT OF COMPOST AND SOME STIMULTORY SUBSTANCES ON GLADIOLUS PLANT \\ A. VEGETATIVE GROWTH AND FLOWERING CHARACTERS
}

\author{
M.A.H. Abdou, M.K. Aly, A.A. El-Sayed, A.R. Khalil and T.A. Helmy \\ Horticulture Department, Faculty of Agriculture, Minia University, Egypt
}

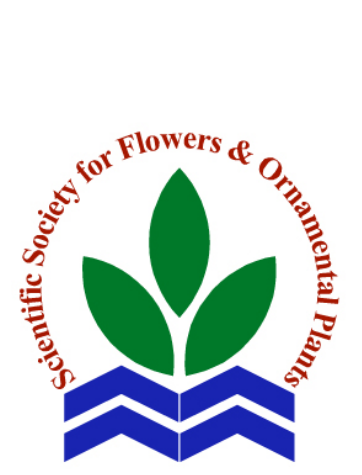

ABSTRACT: The present study was conducted in $2017 / 2018$ and $2018 / 2019$ seasons in the Nursery and Laboratory of Ornamental Plants, Faculty of Agriculture, Minia University to study the effect of compost $(7.5,10.0$ and 12.5 ton/fed) and seven treatments of stimulants i.e. control, seaweeds extract (300 ppm), amino acids (200 ppm), ascorbic acid (100 ppm), salicylic acid (50 ppm), active yeast (5 $\mathrm{g} / \mathrm{l})$ and Minia azotein (50 $\mathrm{ml} / \mathrm{plant})$, as well as their interaction on vegetative growth and flowering parameters of gladiolus plants. Data resulted that all compost levels increased vegetative growth parameters (leaf length, number of leaves/plant and leaves dry weight), flowering Scientific J. Flowers \& aspects (spike length, spike diameter, spike fresh weight, number of Ornamental Plants, florets/plant, lower floret diameter and single floret fresh weight) 8(1):65-74 (2021). comparing with control. Compost at 12.5 ton/fed was the most effective in this concern. All six used treatments of stimulant

Received:

$3 / 12 / 2020$

Accepted:

$25 / 12 / 2020$

Corresponding author: M.A.H. Abdou

mahmoud.abdo@mu.edu.eg Key words: Gladiolus, compost, seaweeds extract, amino acids, ascorbic, salicylic, yeast and Minia azotein.

\section{INTRODUCTION}

Gladiolus is one of the most important ornamental bulbs. It belongs to the Family Iridaceae, it has decorative spike which carries numerous florets. Its flowers are excellent attractive cut flowers, which are needed for local markets in winter and spring, as well as, for export to foreign ones.

Compost as organic fertilizer improves soil texture, increases ion exchange capacity of the soil, increases buffering capacity and adsorbs essential nutrients against leaching and increases microorganisms (McHoy, 2000; Paulin and Omalley, 2008). Many authors concluded that compost as an organic fertilizer increased vegetative growth traits, flowering characters and corms and cormels productivity of gladiolus plant such as Ahmed (2013), Abdou and Ibrahim (2015), Khalil (2015), Abdou et al. (2018) and Hassan and Abd El-Azeim (2020).

The effect of stimulatory substances on improving plant growth, flowering was emphasized by De lunica and Vecchietti (2012) and Hassan (2016) on gladiolus plants Regarding seaweeds extract; Abd ElAziz et al. (2009), Sewedan et al. (2012) and Hashish et al. (2015) on gladiolus plants and regarding amino acids; Abdou et al. (2004), El-Sayed (2004) and El-Deeb (2016) on gladiolus plants.

Concerning N-fixing bacteria; moreover, Sajjad et al. (2014), Khalil (2015), Pal et al. (2015), Pansuriya et al. (2018) and Hassan 
and Abd El-Azeim (2020) cleared that salicylic acid treatment increased growth of gladiolus plants. Also, Abd El-Aziz et al. (2009), Abo Leila and Eid (2011) and Abdou et al. (2019), on gladiolus, showed that all vegetative growth traits were increased due to ascorbic acid treatment.

Therefore, the present work aimed to study the effect of compost and some stimulatory substances on vegetative growth parameters and flowering traits of gladiolus cv. Eurovisin.

\section{MATERIALS AND METHODS}

This field experiment was carried out during the two successive seasons of 2017/2018 and 2018/2019 at the Nursery of Ornamental plants, Faculty of Agriculture, Minia University to investigate the effect of compost, and some stimulatory substances, as well as, their interactions on the vegetative growth and flowering of gladiolus (Gladiolus grandiflorus, cv. Eurovision) plants.

The corms of Gladiolus grandiflorus, cv. Eurovision were obtained from Holland by Basiouny Nurseries, Cairo, Egypt. Average corm's diameter was 3.0 and $3.3 \mathrm{~cm}$ and average corm's weight was 9.9 and $10.4 \mathrm{~g}$ for both seasons, respectively, corms were soaked in Pinlate at $1.0 \mathrm{~g} / \mathrm{l}$ for one minute before planting in both seasons. The experiment was arranged in a randomized complete block design in a split plot design with three replicates. The main plot (A) included four levels of compost 0.0, 7.5, 10.0 and $12.5 \mathrm{ton} / \mathrm{fed}$ ), while seven treatments of stimulants occupied the sub-plot (B), namely, control, seaweeds extract (300 ppm), amino acids (200 ppm), ascorbic acid (100 ppm), salicylic acid (50 ppm), active yeast $(5 \mathrm{~g} / \mathrm{l})$ and Minia azotein $(50 \mathrm{ml} /$ plant $)$. Therefore, the interaction treatments $(\mathrm{A} \times \mathrm{B})$ were 28 treatments. The experimental unit (plot) was $1.5 \times 1.8 \mathrm{~m}$. containing 3 ridges, $50 \mathrm{~cm}$ apart. Corms were planted on September 1 st for both seasons in hills, 15 $\mathrm{cm}$ apart (12 corms/ridge). The physical and chemical analysis of the used soil in both seasons were determined according to Page et al. (1982) and shown in Table (a).

Compost (plant residues) was obtained from Egypt Company for Circulate Solid Residues at El-Minia New City and added during preparing the soil to cultivation in both seasons. The chemical analysis of compost is shown in Table (b).

Minia azotein (containing $\mathrm{N}$-fixing bacteria) was obtained from the Laboratory of Bio-fertilizers, Genetic Department, Faculty of Agriculture, Minia University. It was applied at $50 \mathrm{ml} /$ plant three times to the soil around each plant, one month and two months from planting date, and after flowers cut.

The dry matter of yeast (Saccharomyces cerevisia), was $95 \%$ and live cells were 11.6 x 109/g. The yeast suspension was prepared by dissolving dry yeast and sugar together (ratio of $1: 1, \mathrm{w} / \mathrm{w}$ basic) in warm water (38 Co) and let it to stand for two hours before spraying to enhance yeast activity (Skoog and Miller, 1957), chemical analysis of the dry yeast is presented in Table (c).

Algeser product contains seaweeds extract, which was obtained from Shoura Chemical Company, Cairo-Alexandria Desert Road, Giza Governorate, Egypt. The chemical properties of the used seaweeds extract were listed in Table (d).

Aminoactal product contains a mixture of three amino acids (treptophan, methionine and cysteine), which was obtained from Shoura Chemical Company, CairoAlexandria Desert Road, Giza Governorate, Egypt.

The seaweeds extract, amino acids, ascorbic acid, salicylic acid and yeast were applied by hand spraying till run off, three times on the same schedule of Minia azotein bio-fertilizer. The agricultural practices were performed as usual in the cultivation region. 
Table a. Physical and chemical properties of the used soil during the two seasons of 2017/2018 and 2018/2019.

\begin{tabular}{|c|c|c|c|c|c|}
\hline \multirow{2}{*}{ Soil character } & \multicolumn{2}{|c|}{ Values } & \multirow{2}{*}{$\begin{array}{c}\text { Soil } \\
\text { character }\end{array}$} & \multicolumn{2}{|c|}{ Values } \\
\hline & $2017 / 2018$ & 2018/2019 & & $2017 / 2018$ & 2018/2019 \\
\hline \multicolumn{3}{|c|}{ Physical properties } & \multicolumn{3}{|c|}{$\begin{array}{l}\text { Exchangeable nutrients } \\
\end{array}$} \\
\hline Sand $(\%)$ & 28.98 & 28.90 & $\mathrm{Ca}^{++}(\mathrm{mg} / 100 \mathrm{~g}$ soil $)$ & 31.12 & 31.43 \\
\hline Silt (\%) & 29.87 & 30.64 & $\mathrm{Mg}^{++}(\mathrm{mg} / 100 \mathrm{~g}$ soil $)$ & 3.51 & 3.56 \\
\hline Clay $(\%)$ & 41.15 & 40.46 & $\mathrm{Na}^{+}(\mathrm{mg} / 100 \mathrm{~g}$ soil $)$ & 2.51 & 2.50 \\
\hline Soil type & Clay loam & Clay loam & $\mathrm{K}^{+}(\mathrm{mg} / 100 \mathrm{~g}$ soil $)$ & 2.85 & 2.64 \\
\hline \multicolumn{3}{|c|}{ Chemical properties } & \multicolumn{3}{|c|}{ DTPA-Extractable nutrients } \\
\hline $\mathrm{pH}(1: 2.5)$ & 7.75 & 7.71 & $\mathrm{Fe}(\mathrm{ppm})$ & 8.23 & 8.11 \\
\hline E.C. $(\mathrm{dS} / \mathrm{m})$ & 1.08 & 1.06 & $\mathrm{Cu}(\mathrm{ppm})$ & 2.01 & 2.00 \\
\hline O.M. & 1.54 & 1.59 & $\mathrm{Zn}(\mathrm{ppm})$ & 2.87 & 2.89 \\
\hline $\mathrm{CaCO}_{3}$ & 2.11 & 2.10 & $\mathrm{Mn}(\mathrm{ppm})$ & 8.11 & 8.15 \\
\hline
\end{tabular}

Table b. Chemical analysis of the used compost in both seasons of 2017/2018 and 2018/2019.

\begin{tabular}{lclc}
\hline Properties & Values & Properties & Values \\
\hline Organic carbon (\%) & 25.1 & Total P (\%) & 0.5 \\
Humidity (\%) & 25.0 & Total K (\%) & 1.0 \\
Organic matter & 44.0 & Fe (ppm) & 1750 \\
C/N ratio & 17.5 & Zn (ppm) & 60 \\
pH $(1: 2.5)$ & 8.0 & $\mathrm{Mn} \mathrm{(ppm)}$ & 125 \\
E.C. $(\mathrm{m}$. mhos/cm.) & 5.0 & $\mathrm{Cu}(\mathrm{ppm})$ & 200 \\
Total N $(\%)$ & 1.5 & & \\
\hline
\end{tabular}

Table c. Chemical analysis of the used active dry yeast in both seasons of 2017/2018 and 2018/2019.

\begin{tabular}{ccccc}
\hline Protein (\%) & Ash (\%) & Glycogen (\%) & Fat (\%) & Cellulose (\%) \\
\hline 34.87 & 7.55 & 6.54 & 2.09 & 4.92 \\
\hline
\end{tabular}

Table d. Chemical properties of the used seaweeds extract in both seasons of 2017/2018 and 2018/2019.

\begin{tabular}{lclclc}
\hline \multicolumn{1}{c}{ Character } & Value & \multicolumn{1}{c}{ Character } & Value & Character & Value \\
\hline Moisture \% & 6.0 & $\mathrm{P} \%$ & $0.02-0.09$ & Zn ppm & $10-00$ \\
Organic matter \% & $45-60$ & $\mathrm{~K} \%$ & $1.0-1.2$ & $\mathrm{~B} \mathrm{ppm}$ & $20-100$ \\
Inorganic matter \% & $45-60$ & $\mathrm{Ca} \%$ & $0.2-1.5$ & Mo ppm & $1-5$ \\
Protein \% & $6-8$ & $\mathrm{~S} \mathrm{\%}$ & $3-9$ & Cytokinins \% & 0.02 \\
Carbohydrate \% & $35-50$ & $\mathrm{Mg} \%$ & $0.5-0.9$ & $\mathrm{IAA} \%$ & 0.03 \\
Aliginic acids \% & $10-20$ & $\mathrm{Cu} \mathrm{ppm}$ & $1.0-6.0$ & $\mathrm{ABA} \%$ & 0.01 \\
Mannitol \% & $4-7$ & $\mathrm{Fe} \mathrm{ppm}$ & $50-200$ & & \\
Total N \% & $1.0-1.5$ & $\mathrm{Mn} \mathrm{ppm}$ & $5-12$ & & \\
\hline
\end{tabular}

The following data were recorded:

1. Vegetative growth characters were recorded just before flowering such as, leaf length $(\mathrm{cm})$, number of leaves/plant, and dry weight of leaves/plant (g).

2. Flowering characters were recorded spike length $(\mathrm{cm})$, spike diameter $(\mathrm{mm})$ "under the lower floret", spike fresh weight $(\mathrm{g})$, number of florets/spike, lower floret diameter $(\mathrm{cm})$ and lower floret fresh weight $(\mathrm{g})$.

\section{Statistical analysis:}

Data of the experiment were subjected to the statistically analyzed of variance using MSTAT-C (1986) and LSD test at 5\% was followed to compare between the means of treatments. 


\section{RESULTS AND DISCUSSION}

\section{Vegetative growth parameters:}

Data presented in Table (1) indicated that the leaf length, leaf number and leaves dry weight per plant were significantly increased due to fertilizing plants with 7.5, 10.0 and 12.5 ton/fed compost comparing with control. The increase in such three characters was parallel to the increase in the compost level. The highest values were obtained with fertilizing gladiolus by compost at 12.5 ton/fed. The increase in vegetative growth resulting from using compost may be due to improving soil physical, chemical and biological properties including water retention capacity, drainage, $\mathrm{pH}$, availability of nutrients from compost (Zheljazk and Warman, 2004 and Griffin and Hutchinson, 2007). Similar results were recorded by Ahmed (2013), Abdou and Ibrahim (2015), Khalil (2015), Hassan (2016), and Hassan and Abd El-Azeim (2020) on gladiolus plants.

Regarding the effect of stimulatory substances, data present in Table (1) showed that all six used treatments (seaweeds extract, amino acids, salicylic acid, ascorbic acid, active yeast and Minia azotein) significantly increased all vegetative growth traits (leaf length, number of leaves/plant and dry weight of leaves/plant) as compared to control. Amino acids followed by active yeast treatments were the most effective than other treatments in this concern. Amino acids can affect plant growth and development through their influence on IAA biosynthesis, protein assimilation which are necessary for cell formation and consequently increase in plant growth (Phillips, 1971; Russel, 1982 and Walter and Nawacki, 1987).

The interaction between the experimental factors (A x B) was significant for all plant growth traits i.e. leaf length, number of leaves/plant and dry weight of leaves/plant, in both seasons. The highest values were obtained due to supplying the soil of gladiolus plants with 12.5 ton/fed compost in combination with amino acids followed by 12.5 ton/fed compost with active yeast.

Similarly, Ahmed (2019) on gladiolus, regarding the best interaction between compost and amino acid, and Ahmed (2013) on gladiolus, concerning the effect of interaction between compost and active yeast.

\section{Flowering parameters:}

Data presented in Tables (2 and 3) revealed that all compost level treatments led to significant increases in spike length, spike diameter, spike fresh weight, number of florets/spike, lower floret diameter and lower floret fresh weight as compared to unfertilized plants in both seasons. The maximum values were obtained by using 12.5 ton/fed compost.

These results are in harmony with the findings of Ahmed (2013), Abdou and Ibrahim (2015), Khalil (2015), Hassan (2016), and Hassan and Abd El-Azeim (2020) on gladiolus plants. Compost treatments (Table, 1) simulative the different vegetative growth, that directly reflected on various flowering aspects (Niazian and Nalousi, 2020).

Concerning sub plots treatments, data presented in Tables ( 2 and 3 ) showed that all used six treatments considerably enhanced flowering parameters i.e. spike length, spike diameter, spike fresh weight, number of florets/spike, lower florets diameter and lower floret fresh weight as compared to control plants. The highest values were obtained by using amino acids followed by active yeast.

In harmony with these results concerning amino acids, those were obtained by Sewedan et al. (2012), Ahmed (2013), Abdou and Ibrahim (2015), Hashish et al. (2015), Hassan (2016) and Khattab et al. (2016) on gladiolus plants. Moreover, ElNaggar and Sweedan (2009) on Hippeastrum vittatum and Afifipour and Kosh-Khui (2015) on Pulianthes tuberose, who 
Table 1. Effect of compost, some stimulatory substances and their combination on leaf length, leaf number and leaves dry weight/plant of gladiolus cv. Eurovisin during 2017/2018 and 2018/2019 seasons.

\begin{tabular}{|c|c|c|c|c|c|c|c|c|c|c|}
\hline \multirow{3}{*}{$\begin{array}{c}\text { Stimulatory substances } \\
\text { treatment (B) }\end{array}$} & \multicolumn{10}{|c|}{ Compost levels (ton/feddan) (A) } \\
\hline & $\mathbf{0}$ & 7.5 & 10.0 & 12.5 & $\begin{array}{c}\text { Mean } \\
\text { (B) }\end{array}$ & $\mathbf{0}$ & 7.5 & 10.0 & $12 / 5$ & $\begin{array}{c}\text { Mean } \\
\text { (B) }\end{array}$ \\
\hline & \multicolumn{5}{|c|}{ The $1^{\text {st }}$ season $(2017 / 2018)$} & \multicolumn{5}{|c|}{ The $2^{\text {nd }}$ season $(2018 / 2019)$} \\
\hline & \multicolumn{10}{|c|}{ Leaf length (cm) } \\
\hline Control & 33.1 & 35.2 & 35.4 & 35.7 & 34.9 & 33.5 & 34.0 & 35.0 & 36.3 & 34.7 \\
\hline Seaweed extract, 300 ppm & 33.7 & 36.5 & 36.8 & 37.3 & 36.1 & 33.5 & 36.8 & 36.8 & 37.4 & 36.1 \\
\hline Amino acids, 200 ppm & 34.0 & 37.1 & 37.4 & 37.8 & 36.6 & 34.4 & 37.3 & 37.8 & 38.2 & 36.9 \\
\hline Ascorbic acids, 100 ppm & 33.5 & 35.8 & 36.0 & 36.4 & 35.4 & 33.4 & 35.8 & 36.4 & 36.8 & 35.6 \\
\hline Salicylic acids, 100 ppm & 33.4 & 35.9 & 36.3 & 36.8 & 35.6 & 33.5 & 36.0 & 36.5 & 36.9 & 35.7 \\
\hline Active yeast, 5 g/l & 33.9 & 36.8 & 37.1 & 37.4 & 36.3 & 33.6 & 37.0 & 37.1 & 37.6 & 36.3 \\
\hline Minia azotein, $50 \mathrm{ml}$ & 33.6 & 36.4 & 36.7 & 37.2 & 36.0 & 33.5 & 36.6 & 36.7 & 37.3 & 36.0 \\
\hline Mean (A) & 33.6 & 36.2 & 36.5 & 36.9 & & 33.7 & 36.2 & 36.6 & 37.2 & \\
\hline \multirow[t]{2}{*}{ L.S.D. at $5 \%$} & \multicolumn{2}{|c|}{ A: 0.3} & B: 0.2 & \multicolumn{2}{|c|}{ AB: 0.4} & \multicolumn{2}{|c|}{ A: 0.4} & B: 0.2 & \multicolumn{2}{|c|}{ AB: 0.6} \\
\hline & \multicolumn{10}{|c|}{ Leaf number/plant } \\
\hline Control & 8.1 & 8.3 & 8.7 & 8.9 & 8.5 & 7.8 & 8.3 & 8.8 & 9.0 & 8.5 \\
\hline Seaweed extract, 300 ppm & 8.6 & 8.8 & 8.9 & 9.5 & 9.0 & 8.1 & 9.0 & 9.4 & 10.0 & 9.1 \\
\hline Amino acids, 200 ppm & 9.0 & 9.2 & 9.7 & 10.0 & 9.5 & 8.1 & 9.5 & 9.8 & 10.4 & 9.5 \\
\hline Ascorbic acids, 100 ppm & 8.4 & 8.6 & 8.9 & 9.0 & 8.4 & 7.9 & 8.6 & 8.9 & 9.4 & 8.7 \\
\hline Salicylic acids, 100 ppm & 8.6 & 8.8 & 9.0 & 9.1 & 8.9 & 8.0 & 8.7 & 9.0 & 9.6 & 8.9 \\
\hline Active yeast, $5 \mathrm{~g} / \mathrm{l}$ & 8.4 & 9.2 & 9.3 & 9.7 & 9.2 & 8.0 & 9.2 & 9.6 & 10.2 & 9.3 \\
\hline Minia azotein, $50 \mathrm{ml}$ & 8.7 & 8.9 & 9.1 & 9.2 & 9.0 & 8.0 & 8.9 & 9.2 & 9.8 & 8.0 \\
\hline Mean (A) & 8.5 & 8.8 & 9.1 & 9.3 & & 8.0 & 8.9 & 9.2 & 9.8 & \\
\hline \multirow[t]{2}{*}{ L.S.D. at $5 \%$} & \multicolumn{2}{|c|}{ A: 0.1} & B: 0.2 & \multicolumn{2}{|c|}{ AB: 0.4} & \multicolumn{2}{|c|}{ A: 0.3} & B: 0.2 & \multicolumn{2}{|c|}{ AB: 0.4} \\
\hline & \multicolumn{9}{|c|}{ Leaves dry weight/plant (g) } & \\
\hline Control & 3.69 & 3.78 & 3.96 & 4.14 & 3.85 & 3.75 & 3.99 & 4.23 & 4.34 & 4.08 \\
\hline Seaweed extract, 300 ppm & 4.41 & 4.51 & 4.56 & 4.87 & 4.59 & 4.55 & 5.05 & 5.28 & 5.61 & 5.13 \\
\hline Amino acids, 200 ppm & 5.15 & 5.18 & 5.48 & 5.75 & 5.39 & 5.19 & 6.11 & 6.29 & 6.71 & 6.08 \\
\hline Ascorbic acids, 100 ppm & 3.84 & 3.93 & 4.07 & 4.12 & 3.99 & 3.78 & 4.12 & 4.26 & 4.50 & 4.17 \\
\hline Salicylic acids, 100 ppm & 3.94 & 4.03 & 4.13 & 4.19 & 4.07 & 3.85 & 4.19 & 4.34 & 4.62 & 4.25 \\
\hline Active yeast, 5 g/l & 4.52 & 4.94 & 5.04 & 5.23 & 4.93 & 4.90 & 5.63 & 5.88 & 6.24 & 4.66 \\
\hline Minia azotein, $50 \mathrm{ml}$ & 4.06 & 4.13 & 4.72 & 4.29 & 4.18 & 4.31 & 4.78 & 4.94 & 5.26 & 4.82 \\
\hline Mean (A) & 4.23 & 4.35 & 4.49 & 4.66 & & 4.33 & 4.84 & 4.97 & 5.33 & \\
\hline L.S.D. at $5 \%$ & \multicolumn{2}{|c|}{ A: 0.11} & B: 0.14 & \multicolumn{2}{|c|}{ AB: 0.28} & \multicolumn{2}{|c|}{ A: 0.12} & B: 0.09 & \multicolumn{2}{|c|}{ AB: 0.70} \\
\hline
\end{tabular}


Table 2. Effect of compost, some stimulatory substances and their combination on spike length, spike diameter and spike fresh weight of gladiolus cv. Eurovisin during 2017/2018 and 2018/2019 seasons.

\begin{tabular}{|c|c|c|c|c|c|c|c|c|c|c|}
\hline \multirow{3}{*}{$\begin{array}{c}\text { Stimulatory substances } \\
\text { treatment (B) }\end{array}$} & \multicolumn{10}{|c|}{ Compost levels (ton/feddan) (A) } \\
\hline & $\mathbf{0}$ & 7.5 & 10.0 & 12.5 & $\begin{array}{c}\text { Mean } \\
\text { (B) }\end{array}$ & $\mathbf{0}$ & 7.5 & 10.0 & $12 / 5$ & $\begin{array}{c}\text { Mean } \\
\text { (B) }\end{array}$ \\
\hline & \multicolumn{5}{|c|}{ The $1^{\text {st }}$ season $(2017 / 2018)$} & \multicolumn{5}{|c|}{ The $2^{\text {nd }}$ season $(2018 / 2019)$} \\
\hline & \multicolumn{10}{|c|}{ Spike length $(\mathrm{cm})$} \\
\hline Control & 55.30 & 61.00 & 62.60 & 65.20 & 61.02 & 58.41 & 61.91 & 62.88 & 66.41 & 63.40 \\
\hline Seaweed extract, 300 ppm & 63.56 & 70.41 & 73.35 & 76.81 & 71.03 & 66.90 & 71.55 & 73.46 & 77.70 & 72.40 \\
\hline Amino acids, 200 ppm & 66.63 & 72.55 & 78.35 & 82.98 & 75.13 & 69.71 & 75.55 & 78.44 & 83.55 & 76.81 \\
\hline Ascorbic acids, 100 ppm & 59.61 & 65.82 & 67.55 & 69.91 & 65.72 & 62.92 & 66.42 & 67.38 & 70.96 & 66.92 \\
\hline Salicylic acids, 100 ppm & 60.73 & 67.71 & 70.45 & 73.88 & 68.21 & 63.95 & 68.53 & 70.45 & 74.68 & 69.40 \\
\hline Active yeast, 5 g/l & 65.55 & 70.45 & 75.23 & 78.91 & 72.54 & 68.80 & 73.41 & 75.33 & 79.50 & 74.26 \\
\hline Minia azotein, $50 \mathrm{ml}$ & 61.95 & 68.91 & 71.95 & 71.38 & 69.55 & 65.44 & 70.04 & 71.95 & 76.18 & 70.90 \\
\hline Mean (A) & 61.91 & 68.12 & 71.36 & 74.72 & & 65.16 & 69.63 & 71.41 & 75.57 & \\
\hline \multirow[t]{2}{*}{ L.S.D. at $5 \%$} & \multicolumn{2}{|c|}{ A: 2.80} & B: 2.04 & \multicolumn{2}{|c|}{$\mathrm{AB}: 4.04$} & \multicolumn{2}{|c|}{ A: 1.78} & B: 2.03 & \multicolumn{2}{|c|}{ AB: 4.06} \\
\hline & \multicolumn{10}{|c|}{ Spike diameter (mm) } \\
\hline Control & 7.8 & 8.6 & 9.5 & 10.4 & 9.1 & 7.9 & 8.7 & 9.6 & 10.6 & 9.2 \\
\hline Seaweed extract, 300 ppm & 10.6 & 12.4 & 13.3 & 16.4 & 13.2 & 10.5 & 12.5 & 14.9 & 16.9 & 13.7 \\
\hline Amino acids, 200 ppm & 11.8 & 13.7 & 14.7 & 17.9 & 14.5 & 11.7 & 13.7 & 15.4 & 18.4 & 14.8 \\
\hline Ascorbic acids, 100 ppm & 8.9 & 10.6 & 12.4 & 14.3 & 11.6 & 8.9 & 10.6 & 12.5 & 14.5 & 11.6 \\
\hline Salicylic acids, 100 ppm & 9.5 & 11.3 & 13.2 & 15.2 & 12.3 & 9.5 & 11.4 & 13.6 & 15.6 & 12.5 \\
\hline Active yeast, $5 \mathrm{~g} / \mathrm{l}$ & 11.3 & 13.1 & 14.0 & 17.1 & 13.9 & 11.5 & 13.1 & 15.7 & 17.6 & 14.4 \\
\hline Minia azotein, $50 \mathrm{ml}$ & 10.0 & 11.8 & 13.7 & 15.8 & 12.8 & 10.1 & 11.9 & 14.3 & 16.2 & 13.1 \\
\hline Mean (A) & 10.0 & 10.4 & 13.0 & 14.4 & & 10.0 & 11.7 & 13.7 & 15.7 & \\
\hline \multirow[t]{2}{*}{ L.S.D. at $5 \%$} & \multicolumn{2}{|c|}{ A: 0.4} & B: 0.6 & \multicolumn{2}{|c|}{ AB: 1.2} & \multicolumn{2}{|c|}{ A: 0.8} & B: 0.4 & \multicolumn{2}{|c|}{ AB: 0.8} \\
\hline & \multicolumn{10}{|c|}{ Spike fresh weight (g) } \\
\hline Control & 16.65 & 18.46 & 18.84 & 19.63 & 18.37 & 18.17 & 19.25 & 19.56 & 20.65 & 19.41 \\
\hline Seaweed extract, 300 ppm & 19.39 & 21.48 & 22.37 & 23.43 & 21.67 & 21.21 & 22.68 & 23.29 & 24.63 & 22.95 \\
\hline Amino acids, 200 ppm & 20.40 & 22.20 & 23.98 & 25.39 & 22.99 & 22.17 & 24.02 & 24.94 & 26.65 & 24.42 \\
\hline Ascorbic acids, 100 ppm & 18.12 & 20.01 & 20.54 & 21.25 & 19.98 & 19.88 & 20.99 & 21.29 & 22.42 & 21.15 \\
\hline Salicylic acids, 100 ppm & 20.05 & 20.65 & 21.49 & 22.51 & 21.18 & 20.27 & 21.72 & 22.33 & 23.67 & 22.00 \\
\hline Active yeast, 5 g/l & 20.06 & 21.56 & 23.03 & 24.15 & 22.20 & 21.88 & 23.34 & 23.96 & 25.30 & 23.62 \\
\hline Minia azotein, $50 \mathrm{ml}$ & 18.90 & 20.95 & 21.95 & 22.99 & 21.20 & 20.74 & 22.20 & 22.81 & 24.15 & 22.39 \\
\hline Mean (A) & 19.08 & 20.75 & 21.74 & 22.76 & & 20.62 & 22.03 & 22.60 & 23.91 & \\
\hline L.S.D. at $5 \%$ & A: 0. & & B: 0.64 & $\mathrm{AB}$ & 0.28 & A: 0 . & & B: 0.65 & $\mathrm{AB}$ & : 1.30 \\
\hline
\end{tabular}


Table 3. Effect of compost, some stimulatory substances and their combination on number of florets/spike, lower florets diameter and lower floret fresh weight of gladiolus cv. Eurovisin during 2017/2018 and 2018/2019 seasons.

\begin{tabular}{|c|c|c|c|c|c|c|c|c|c|c|}
\hline \multirow{3}{*}{$\begin{array}{l}\text { Stimulant substances } \\
\text { treatments (B) }\end{array}$} & \multicolumn{10}{|c|}{ Compost levels (ton/feddan) (A) } \\
\hline & $\mathbf{0}$ & 7.5 & 10.0 & 12.5 & $\begin{array}{c}\text { Mean } \\
\text { (B) }\end{array}$ & $\mathbf{0}$ & 7.5 & 10.0 & $12 / 5$ & $\begin{array}{l}\text { Mean } \\
\text { (B) }\end{array}$ \\
\hline & \multicolumn{5}{|c|}{ The $1^{\text {st }}$ season $(2017 / 2018)$} & \multicolumn{5}{|c|}{ The $2^{\text {nd }} \operatorname{season}(2018 / 2019)$} \\
\hline & \multicolumn{10}{|c|}{ Number of florets/spike } \\
\hline Control & 9.33 & 11.35 & 11.75 & 12.50 & 11.24 & 11.17 & 11.33 & 12.17 & 12.33 & 11.75 \\
\hline Seaweed extract, $300 \mathrm{ppm}$ & 11.85 & 13.00 & 13.25 & 13.43 & 12.88 & 12.80 & 13.35 & 13.59 & 13.98 & 13.43 \\
\hline Amino acids, 200 ppm & 12.25 & 13.11 & 13.17 & 14.50 & 13.26 & 13.25 & 13.77 & 13.95 & 14.21 & 13.79 \\
\hline Ascorbic acids, 100 ppm & 11.61 & 11.64 & 12.44 & 12.89 & 12.15 & 12.00 & 12.19 & 12.50 & 12.50 & 12.30 \\
\hline Salicylic acids, 100 ppm & 11.53 & 11.83 & 12.45 & 13.19 & 12.25 & 12.06 & 12.20 & 12.80 & 13.17 & 12.56 \\
\hline Active yeast, $5 \mathrm{~g} / \mathrm{l}$ & 11.88 & 13.10 & 13.23 & 13.45 & 12.92 & 13.00 & 13.55 & 13.79 & 14.10 & 13.61 \\
\hline Minia azotein, $50 \mathrm{ml}$ & 11.60 & 11.86 & 12.72 & 13.50 & 12.42 & 12.10 & 12.65 & 12.89 & 13.27 & 12.73 \\
\hline Mean (A) & 11.44 & 12.27 & 12.72 & 13.34 & & 12.34 & 12.72 & 13.10 & 13.37 & \\
\hline \multirow[t]{2}{*}{ L.S.D. at $5 \%$} & \multicolumn{2}{|c|}{ A: 0.32} & B: 0.30 & \multicolumn{2}{|c|}{ AB: 0.60} & \multicolumn{2}{|c|}{ A: 0.36} & B: 0.25 & \multicolumn{2}{|c|}{ AB: 0.50} \\
\hline & \multicolumn{10}{|c|}{ Lower floret diameter (mm) } \\
\hline Control & 6.25 & 6.76 & 7.04 & 7.63 & 6.82 & 6.18 & 6.53 & 7.39 & 7.77 & 6.97 \\
\hline Seaweed extract, $300 \mathrm{ppm}$ & 6.65 & 7.15 & 7.44 & 8.04 & 7.32 & 6.62 & 6.98 & 7.79 & 8.20 & 7.40 \\
\hline Amino acids, 200 ppm & 6.77 & 7.29 & 7.50 & 8.39 & 7.47 & 6.76 & 7.14 & 7.93 & 8.48 & 7.58 \\
\hline Ascorbic acids, 100 ppm & 6.46 & 6.95 & 7.24 & 7.83 & 7.12 & 6.39 & 6.74 & 7.59 & 7.99 & 7.19 \\
\hline Salicylic acids, 100 ppm & 6.57 & 7.05 & 7.35 & 7.94 & 7.23 & 6.50 & 6.85 & 7.70 & 8.10 & 7.29 \\
\hline Active yeast, $5 \mathrm{~g} / \mathrm{l}$ & 6.71 & 7.21 & 7.50 & 8.11 & 7.18 & 6.68 & 7.04 & 7.85 & 8.28 & 7.46 \\
\hline Minia azotein, $50 \mathrm{ml}$ & 6.59 & 7.05 & 7.38 & 7.98 & 7.26 & 6.55 & 6.90 & 7.73 & 8.14 & 7.33 \\
\hline Mean (A) & 6.57 & 7.07 & 7.35 & 7.99 & & 6.53 & 6.88 & 7.71 & 8.14 & \\
\hline \multirow[t]{2}{*}{ L.S.D. at $5 \%$} & \multicolumn{2}{|c|}{ A: 0.17} & B: 0.14 & \multicolumn{2}{|c|}{ AB: 0.28} & \multicolumn{2}{|c|}{ A: 0.20} & B: 0.11 & \multicolumn{2}{|c|}{ AB: 0.22} \\
\hline & \multicolumn{10}{|c|}{ Lower floret fresh weight (g) } \\
\hline Control & 2.75 & 2.97 & 3.10 & 3.36 & 3.05 & 2.72 & 2.80 & 3.25 & 3.42 & 3.05 \\
\hline Seaweed extract, 300 ppm & 3.05 & 3.28 & 3.43 & 3.70 & 3.37 & 3.00 & 3.21 & 3.62 & 3.88 & 3.43 \\
\hline Amino acids, 200 ppm & 3.21 & 3.36 & 3.63 & 3.92 & 3.53 & 3.18 & 3.41 & 3.85 & 4.12 & 3.64 \\
\hline Ascorbic acids, 100 ppm & 2.87 & 3.09 & 3.23 & 3.48 & 3.17 & 2.88 & 3.03 & 3.42 & 3.60 & 3.23 \\
\hline Salicylic acids, 100 ppm & 2.92 & 3.14 & 3.27 & 3.53 & 3.22 & 2.97 & 3.10 & 3.49 & 3.75 & 3.32 \\
\hline Active yeast, $5 \mathrm{~g} / \mathrm{l}$ & 3.11 & 3.35 & 3.51 & 3.79 & 3.44 & 3.06 & 3.28 & 3.71 & 3.98 & 3.50 \\
\hline Minia azotein, $50 \mathrm{ml}$ & 2.98 & 3.20 & 3.35 & 3.61 & 3.29 & 2.95 & 3.15 & 3.33 & 3.80 & 3.36 \\
\hline Mean (A) & 2.98 & 3.20 & 3.36 & 3.63 & & 2.96 & 3.14 & 3.56 & 3.79 & \\
\hline L.S.D. at $5 \%$ & \multicolumn{2}{|c|}{ A: 0.16} & B: 0.11 & \multicolumn{2}{|c|}{ AB: 0.22} & \multicolumn{2}{|c|}{ A: 0.17} & B: 0.15 & \multicolumn{2}{|c|}{ AB: 0.32} \\
\hline
\end{tabular}


concluded that active yeast had positive effect on flowering aspects.

The interaction between compost and stimulatory substances treatments was significant for all flowering parameters measurement in both seasons. The best interaction treatments were obtained by fertilizing gladiolus with 12.5 ton/fed compost and spraying plants with amino acids or active yeast.

\section{CONCLUSION}

From our results, fertilizing gladiolus with 12.5 ton/fed compost with combination with amino acids led to better vegetative growth along with high quality and quantity of flowering production of Gladiolus grandiflorus, cv. Eurovision plants.

\section{REFERENCES}

Abd El-Aziz, N.G.; Taha, L.S. and Ibrahim, S.M.M. (2009). Some studies on the effect of putrescine, ascorbic acid and thiamine on growth, flowering and some chemical constituents of Gladiolus plants at Nubaria. Ozean J. of Applied Sci., 2(2):169-179.

Abdou, M.A.H. and Ibrahim, T.I.E. (2015). Response of gladiolus cv. Carmen to compost, biofertilization and some vitamin treatments. Proc. the $1^{\text {st }}$ Conf. of SSFOP "Future of Ornamental Plants in Egypt", Cairo, Egypt, Scientific J. Flowers \& Ornamental Plants, 2(1):1-10.

Abdou, M.A.H.; Aly, M.K.; El-Sayed, A.A. and Ahmed, A.S.A. (2019). Influence of organic, mineral, biofertilizers and some vitamin treatments on: A. Vegetative growth and flowering aspects of Gladiolus grandiflorus var. Gold Field plants. Scientific J. Flowers \& Ornamental Plants, 6(2):113-124.

Abdou, M.A.H.; Attia, F.; Aly, M.K. and Sayed, I.H. (2004). Response of gladiolus plants to some bio and chemical fertilization treatments. 1- Vegetative growth and flowering; Proc. The Fifth
Arabian Hort. Con. Ismailia, Egypt, 2428 March, 1:50-62.

Abdou, M.A.H.; Badran, F.S.; Ahmed, E.T.; Taha, R.A. and Abdel-Mola, M.A.M. (2018). Effect of compost and some natural stimulant treatments on: I. Vegetative growth and flowering aspects of Gladiolus grandiflorus cv. Peter Pears plants. Proc. the $4^{\text {th }}$ Conf. of SSFOP "Ornamental Plants and Environment", Cairo, Egypt, Scientific J. Flowers \& Ornamental Plants, 5(2):105-114.

Abo Leila, B. and Eid, R. (2011). Improving gladiolus growth, flower keeping quality by using some vitamins application. Journal of American Science, 7(3):169174.

Afifipour, Z. and Kosh-Khui, M. (2015). Efficacy of spraying a mixture of amino acids on the physiological and morphological characteristics of tuberose (Polianthes tuberosa L.). International J. of Hort. Sci., 2(2):199-204.

Ahmed, A.S.A. (2013). Physiological Studies on Gladiolus Plant. M.Sc. Thesis, Fac. Agric., Minia Univ., Egypt, 188 p.

Ahmed, A.S.A. (2019). Exogenous application of calcium improved the vegetative attributes and corm production in gladiolus. Sarhad Journal of Agriculture, 35(3):1011-1019.

De Luncia, B.D. and Vecchietti, L. (2012). Type of bio-stimulant and application method effects on stem quality and root system growth in L.A. Lily. European J. of Hort. Sci., 77(1):10-15.

El-Deeb, M.B.E.M. (2016). Effect of Mineral and Bio-Fertilization on the Vegetative Growth, Flowering and Corms Production of Gladiolus Plant Growing Under Different Media. Ph.D. Thesis, Fac. Agric., Alex Univ., 165 p.

El-Naggar, A.H. and Sewedan, E.A. (2009). Effect of light intensity and amino acid tryptophan on the growth and flowering of amaryllis (Hippeastrum vittatum, 
Herba.). J. Agric. and Env. Sci., Egypt, $8(1): 22-42$.

El-Sayed, E.H. (2004). Response of Gladiolus Plants to Some Bio and Chemical Fertilization Treatments. Ph.D. Thesis, Fac. Agric., Minia Univ., Egypt, $142 \mathrm{p}$.

Griffin, T. and Hutchinson, M. (2007). Compost maturity effects on nitrogen and carbon mineralization and plant growth. Compost Science \& Utilization, 15:228236.

Hashish, Kh.L.; Eid, R.A.; Kandil, M.M. and Mazhar, A.A.M. (2015). Study on various levels of salinity on some morphological and chemical composition of gladiolus plants by foliar spray with glutathione and thiamine. International J. of Chem. Tech. Res., 9(8):334-341.

Hassan, A.A. (2016). Response of Gladiolus Plant to Some Fertilization and Antioxidant Treatments. Ph.D. Thesis, Fac. Agric., Minia Univ., Egypt, 215 p.

Hassan, A.A. and Abd El-Azeim, M.M. (2020). Impacts of compost, biofertilizer and/or some antioxidant treatments on gladiolus (Gladiolus grandifloras), A. Vegetative growth and flowering aspects. Scientific J. Flowers \& Ornamental plants, 7(3):269-283.

Khalil, A.R.M. (2015). Physiological Studies on Gladiolus Plant. M.Sc. Thesis, Fac. Agric., Minia Univ., Egypt, 146 p.

Khattab, M.; Shehata, A.; El-Saadate, E.A. and El-Hasni, K. (2016). Effect of glycine, methionine and tryptophan on vegetative growth, flowering and corms production of Gladiolus plant. Alex. Sci. Exch. J., 37:647-659.

McHoy, P. (2000). The Gardening Handbook. Whitecap Books Ltd., Canda, $256 \mathrm{p}$.

MSTAT-C (1986). A microcomputer program for the design management and analysis of Agronomic Research
Experiments (version 4.0), Michigan State Univ., U.S.A.

Niazian, M. and Nalousi, A.M. (2020). Artificial polyploidy induction for improvement of ornamental and medicinal plants. Plant Cell, Tissue and Organ Culture, 142:447-469.

Page, A.L.; Miller, R.H. and Keeney, D.R. (1982). Methods of Soil Analysis; 2. Chemical and Microbiological Properties, American Soc. of Agronomy, Madison, Wisconsin, USA, 1159 p.

Pal, V.; Ram, M. and Kumar, M. (2015). Effect of various levels of spacing and salicylic acid treatment on vegetative growth and flowering of gladiolus (Gladiolus grandiflora, L.) cv. White Prosperity. South Asian J. Food Technol. Environ., 1(1):101-104.

Pansuriya, P.B.; Varu, D.K. and Viradia, R.R. (2018). Effect of biostimulants and biofertilizers on growth, flowering and quality of gladiolus (Gladiolus grandiflorus, L.) cv. American Beauty under greenhouse conditions. International Journal of Chemical Studies, 6(2):2191-2196.

Paulin, B. and Omalley, P. (2008). Compost production and use in Horticulture. Dept. of Agric. and Food, Western Australia, Perth, Bulletin No. 4746, 30 p.

Phillips, I.D.J. (1971). Introduction to the Biochemistry and Physiology of Plant Grow Hormones. McGraw-Hill Education, USA, $173 \mathrm{p}$.

Russel, R.S. (1982). Plant Root Systems $1^{\text {st }}$ Ed. McGraw-Hill Education, USA, 298 p.

Sajjad, Y.; Jaskani, M.; Ashraf, M.; Qasim, M. and Ahmad, R. (2014). Response of morphological and physiological growth attributes to foliar application of plant growth regulators in Gladiolus "White Prosperity". Pak. J. Agric. Sci., 51(1):123-129. 


\section{M.A.H. Abdou et al.}

Sewedan, E.; El-Naggar, H. and Osman, A. (2012). Effect of nitrogen and diphenylamine on Gladiolus hybrid cv. Sancerre production. J. of Hort. Sci. and Ornamental Plants, 4(3):267-274.

Skoog, F. and Miller, C. (1957). Biological Action of Growth Substances. Cambridge Univ. Press, USA, 344 p.
Walter, G.R. and Nawacki, E. (1987). Alkaloids Biology Metabolism in Plants. Planum Press., NY, USA, 152 p.

Zheljazk, V.D. and Warman, P.R. (2004). Source-separated municipal solid waste compost application to Swiss chard and basil. J. of Environ. Quality, 33:542-552.

$$
\begin{aligned}
& \text { تأثير الكمبوست وبعض المواد المنشطة على نبات الجلاديولس } \\
& \text { أ. صفات النمو الخضري والزهري } \\
& \text { محمود عبد الهادي حسن عبده، محمد كمال عبد العال علي، أحمد عبدالمنعم السيد، أميرة رجائي خليل، }
\end{aligned}
$$

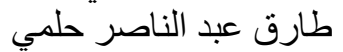

$$
\begin{aligned}
& \text { قسم البساتين، كلية الزر اعة، جامعة المنيا، مصر }
\end{aligned}
$$

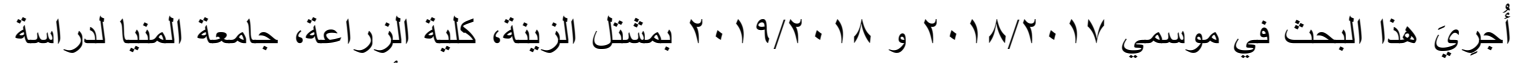

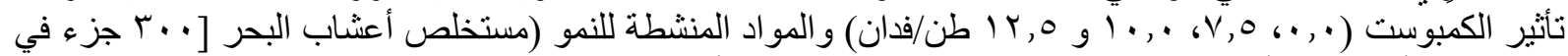

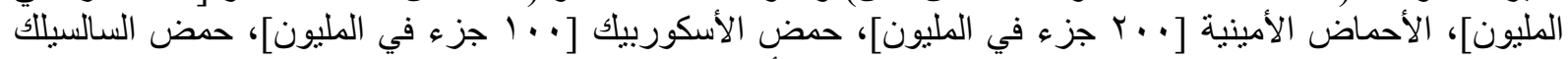

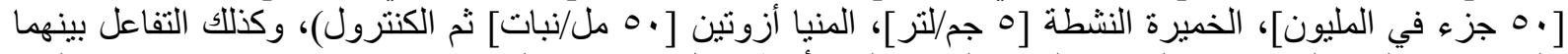

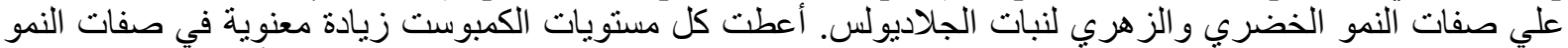

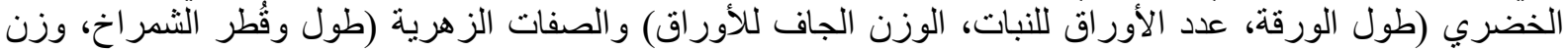

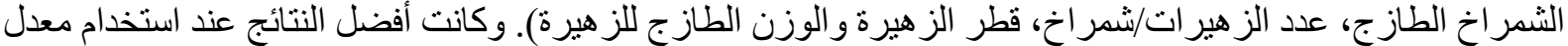

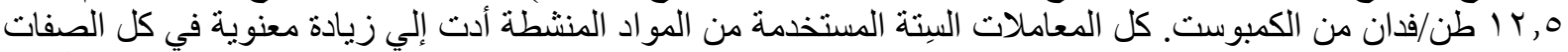

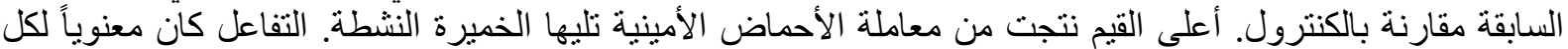

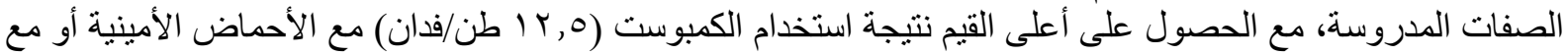
الخمبرة النشطة. 